Revue d'histoire de l'Amérique française

REYUE D.HISTOIRE DE L'AMÉRIQUE FRANÇAISE

\title{
Hagiographie et historiographie
}

En marge d'un livre de Micheline Lachance sur le Frère André

\section{Benoît Lacroix}

Volume 35, numéro 2, septembre 1981

URI : https://id.erudit.org/iderudit/303954ar

DOI : https://doi.org/10.7202/303954ar

Aller au sommaire du numéro

Éditeur(s)

Institut d'histoire de l'Amérique française

ISSN

0035-2357 (imprimé)

1492-1383 (numérique)

Découvrir la revue

Citer cette note

Lacroix, B. (1981). Hagiographie et historiographie : en marge d'un livre de Micheline Lachance sur le Frère André. Revue d'histoire de l'Amérique française, 35(2), 263-267. https://doi.org/10.7202/303954ar d'utilisation que vous pouvez consulter en ligne.

https://apropos.erudit.org/fr/usagers/politique-dutilisation/ 
NOTE CRITIQUE

\title{
HAGIOGRAPHIE ET HISTORIOGRAPHIE
}

\author{
En marge d'un livre de Micheline LACHANCE \\ sur le Frère André
}

Quels peuvent être les mérites d'un tel tête-à-tête avec ce héros populaire du catholicisme traditionnel de l'Amérique française? Disons-le tout de suite: Micheline Lachance n'a pas la méthode pour étudier scientifiquement une mentalité religieuse, représentée ici par celle du Frère André dit autrefois Alfred Bessette (1845-1937); elle n'a pas non plus l'intention de devenir un modèle pour des biographes de stricte observance. Peu de références explicites à ses dossiers, une bibliographie de titres sans mentions d'éditeurs et même de pages. L'importance de cette biographie, sérieuse par ailleurs (cf. Le Frère André, Montréal, les Éditions de l'Homme (1980), 414 p.), tient à d'autres facteurs.

Mi-historienne, mi-journaliste, Micheline Lachance sait surtout, et largement, mettre à profit toutes ses sources visuelles, orales, manuscrites et imprimées: l'iconographie, le Summarium du procès de béatification, de nombreux témoignages d'informateurs encore vivants, dont celui du Père Émile Deguire, etc. La preuve de son honnêteté est que les dates, les lieux, les faits et surtout les personnes en cause peuvent être identifiés par tout lecteur intelligent et soucieux de garanties supplémentaires. Bravant tour à tour le mystère chrétien relié au culte des saints et le merveilleux populaire, allant allègrement d'un témoignage à l'autre, de l'oral à l'imprimé, du visuel au transcrit, elle enchaîne avec une aisance toute gratifiante, nous dirions même avec une joie communicative qui reflète une perception ouverte du sacré en même temps qu'une revalorisation du profane. Rien n'est rejeté a priori, du meilleur ou du pire; les opinions de ses informateurs, fussent-ils les plus dévots ou les plus incrédules, sont respectées; l'irrationnel, le rêve, le fantastique, le miraculeux deviennent aussi dignes d'étude que tout autre aspect plus vérifiable de la réalité vécue par le public. Il n'est plus question de majorer le fait religieux pour l'imposer au lecteur, ni de le réduire à quelque structure préfabriquée. À chaque information qui risquerait d'être trop indiscrète, $M$. L. renvoie délicatement son lecteur, et sans le brusquer, à sa propre liberté de croire ou de ne pas croire. Le merveilleux est absorbé par un récit de vie [263] 
qui laisse transparaitre à la fois le quotidien et l'ordinaire, ce qui a pour effet de rendre plus existentielle et plus réelle «l'histoire de l'obscur portier qui allait accomplir des miracles». "C'était un homme comme tout le monde. Les gens l'aimaient parce qu'il leur ressemblait» (p. 10).

Donc, le lecteur n'est jamais agressé, ni même orienté. Il faut beaucoup de talent et surtout une grande finesse d'esprit pour réussir un exploit pareil dans un milieu où l'hagiographie locale n'a jamais manqué de superlatifs et d'amplificateurs. Ici le dit, le fait révèlent leur sens à mesure. Le Frère André parle, guérit et parfois opère comme un saint. Dans la même personne semblent coexister et le guérisseur et le thaumaturge. Le champ est ouvert, il est libre pour toutes sortes d'hypothèses. On est ramené sans cesse aux récits de la tradition orale et à la foi sécuritaire de l'époque cléricale que l'on sait. Certains liront en souriant - pour ne donner qu'un exemple - ces pages délicieuses et si bien écrites encore sur le Frère André dans ses rapports avec les obsédés de la mode féminine. Tout ceci pour signifier à quel point notre auteur sait faire flèche de tout bois, interroger sans mentir ni profaner, et laisser parler ses informateurs. Elle n'oublie jamais pour autant qu'elle écrit la vie d'un Québécois identifié, populaire, peu lettré, recherché davantage pour ses guérisons-miracles que pour ses conseils pieux, sa piété toute simple, fragile, répétitive comme son humilité, sans parler de sa confiance naïve et pure à saint Joseph le charpentier. Comme elle sait visualiser aussi! Peu de biographes d'ici peuvent, en si peu de mots, réussir autant de portraits et raconter autant de faits qui se lisent comme des romans. Un vrai récital de la mémoire populaire.

«Ça fait que» de sérieux problèmes se posent déjà à nous, historiens du Québec, quant à notre nouvelle manière courte et quelquefois désinvolte de traiter les phénomènes religieux populaires. Micheline Lachance est une femme, une laïque qui ose se pencher sur un dossier habituellement réservé à des prêtres et à des biographes professionnels. L'événement mérite d'être commenté à cause de ses significations futures. En effet, cette façon franche d'aborder le territoire jadis sacré de l'hagiographie traditionnelle est remarquable. Celle-ci, on le sait trop mais il faut le redire, s'est largement illustrée ici par d'innocentes «pieuseries» et par une obéissance toute rituelle à ses modèles européens de la Bonne Presse. Puisque M. L. a su profiter de la largeur de vue des Pères de l'Oratoire Saint-Joseph, puisqu'elle a eu accès à tout ce qu'elle a voulu savoir, le temps ne serait-il pas venu de nous interroger sur ce secteur quelque peu oublié de notre histoire: l'imaginaire religieux des 
Québécois tel que révélé par nos hagiographes de toutes espèces (cf. Un patrimoine méprisé: la religion populaire des Québécois, éd. Jean Simard, René Bouchard et Jocelyne Milot, Montréal, HMH, 1979, 309 p.)?

Non? Oui. Mais à certaines conditions. Pour connaître une mentalité religieuse populaire, pour vérifier jusqu'à quel degré le modèle reçu conduit ou ne conduit pas à des attitudes réelles, pour que toute l'hagiographie d'ici fasse un jour partie de notre univers culturel au mềme titre que l'historiographie, l'archéologie ou la généalogie, il importe en premier lieu qu'elle devienne l'objet d'un corpus ad hoc, qu'elle soit aussitôt resituée dans le contexte d'une étude globale des mentalités et des institutions canadiennesfrançaises.

Ses idéologies elles-mêmes devront être soumises à la critique et non plus seulement énoncées, répétées, ni surtout imposées au lecteur. Bien entendu, l'Église officielle perdra encore quelques plumes dans cette opération critique: perdre des plumes n'est rien si on garde ses ailes. Certains héros sacro-saints de notre histoire populaire le seront moins: il arrive que le sacré gagne là où le merveilleux cède. Un merveilleux mal perçu, mal évalué, fait souvent échec au mystère qui est propre aux phénomènes religieux.

Une étude de ce style exige aussi la plus grande rigueur scientifique, une rigueur qui sait trancher dans le vif des superstitions tout en respectant les requêtes des consciences croyantes. Le phénomène des guérisons et des guérisseurs publics, par exemple, appelle une étude dialectique poussée entre l'univers biologique, la technique, la représentation sociale de la santé (v.g. Santé, médecine et sociologie, actes du Colloque international de sociologie médicale, Paris, CNRS, 1978, 489 p.) et l'univers symbolique du sacré et du culturel. Parler uniquement d'intuition, de logique, de luttes et d'échecs (v.g. Gilles Brunel, "Guérison, magie et symbolique corporelle», dans Anthropologie et sociétés, 4, 2 (1980): 113130), c'est renvoyer le lecteur à l'univers clos des simples guérisseurs. Univers inévitable, bien sûr, mais qui ne rend pas compte de tous les aspects de la guérison, ni surtout de la mentalité religieuse populaire qui la sollicite.

Enfin, tout ce qui se montre excessif dans notre hagiographie populaire, ce que certains appellent d'un terme technique qui risque de créer la confusion du lecteur non initié, la paralittérature religieuse, pourrait devenir un excellent terrain d'échange et de confrontation entre diverses disciplines qui ne sont pas souvent appelées à se rencontrer: histoire, sociologie, anthropologie, ethnologie, psychiatrie, médecine et autres consacrées à des phénomènes semblables. 
Pour notre part, nous rêverions d'un "merveilleux» colloque favorisant ces échanges interdisciplinaires autour du dossier du Frère André. Il ne s'agit pas de promouvoir d'autres guerres de frontières entre universitaires; nous aimerions plutôt, à la manière de la nouvelle histoire (cf. "Histoire aujourd'hui», dans Magazine littéraire, 164 (septembre 1980): 8-44), interroger diversement la même réalité à partir d'un cas limité mais représentatif (à la façon de Le Roy Ladurie), respecter cette réalité, éviter toute domination idéologique à sens unique. Nous dirions même que le texte de $\mathbf{M}$. L. témoigne à sa manière linéaire et rapide d'une largeur de vue capable d'orienter les esprits vers cette histoire globale d'un phénomène de masse.

Reprenons. Le riche corpus déposé à l'Oratoire Saint-Joseph nous paraît comme l'occasion idéale pour étudier la sociologie du milieu, la psychologie du guérisseur et la théologie si courte soitelle d'un thaumaturge, pour relever, comparer plutôt les interprétations, de l'hagiographie courante, enfin pour rappeler le caractère essentiellement merveilleux des premiers récits de guérisons en consultant au besoin l'ethno-psychologie, sans omettre l'histoire des institutions toujours aussi indispensable. Bref, structurer une sorte de biographie sociale et collective du Frère andré serait aussi enrichissant qu'étudier l'événement Lionel Groulx. Le Frère André (1845-1937) résume à lui seul un siècle de religion populaire en Amérique francophone, de même que Lionel Groulx (18781967) représente à sa façon presque un siècle de nationalisme.

D'ailleurs nous ne serions ni les premiers ni les derniers à renouveler l'étude de l'hagiographie, comme à soumettre cette dernière à la critique des sciences humaines. Déjà, et sans oublier les célèbres bollandistes, les preuves sont faites en historiographie française contemporaine avec l'École des Annales, Delumeau, Le Goff, J.-C. Schmitt surtout (cf. Le saint lévrier: Guinefort, guérisseur d'enfants depuis le XIII siècle, Paris, Flammarion, 1979, 273 p., avec les réserves de B. de Gaiffer, dans Analecta Bollandiana, 98, 1980: 440-441) que les méthodes de l'histoire et de l'ethnologie, confrontées à propos de textes hagiographiques ou merveilleux, sont efficaces à révéler comment, entre culture savante et culture populaire, le conflit lui-même est un lieu propice d'interprétation et de compréhension réciproques des interrogations de l'univers humain.

Il ne serait pas si mal aussi que, suivant cet effort de renouvellement de l'hagiographie, les historiens observent collectivement les phénomènes de masse et qu'ils se regroupent pour une étude du vécu quotidien du plus grand nombre jusqu'à redonner la priorité au descriptif, à la res gesta, à l'événement, à sa représentation 
visuelle, orale et finalement textuelle. Une approche moins idéologique de ces représentations et des faits, moins axée en tout cas sur les dualismes trop connus du temporel $v s$ spirituel, de l'exploitant vs l'exploité, un examen plus global du vécu populaire pourraient peut-être à la longue réhabiliter l'historiographie dans tous les milieux et lui rappeler son autonomie traditionnelle ainsi que ses droits de première occupante dans l'univers trouble des sciences humaines.

Pour récapituler et revenir une fois de plus à ce qui nous intéresse présentement, l'étude d'un phénomène autour d'un dossier ouvert au merveilleux, tel celui du Frère André, invite à l'abandon coûte que coûte de la pensée déductiviste, qu'elle s'appelle catholique ou autre. Il devient nécessaire qu'on évite du même coup des encadrements trop mystico-religieux et le rappel des seules idéologies à la mode d'aujourd'hui. Dans cette nouvelle perspective, aucun historien, aucun hagiographe n'est exclu, ni les admirables synthèses déjà fournies par nos maîtres, ni les propos et sujets plus neufs que cette Revue ne cesse d'offrir périodiquement à ses lecteurs. 